
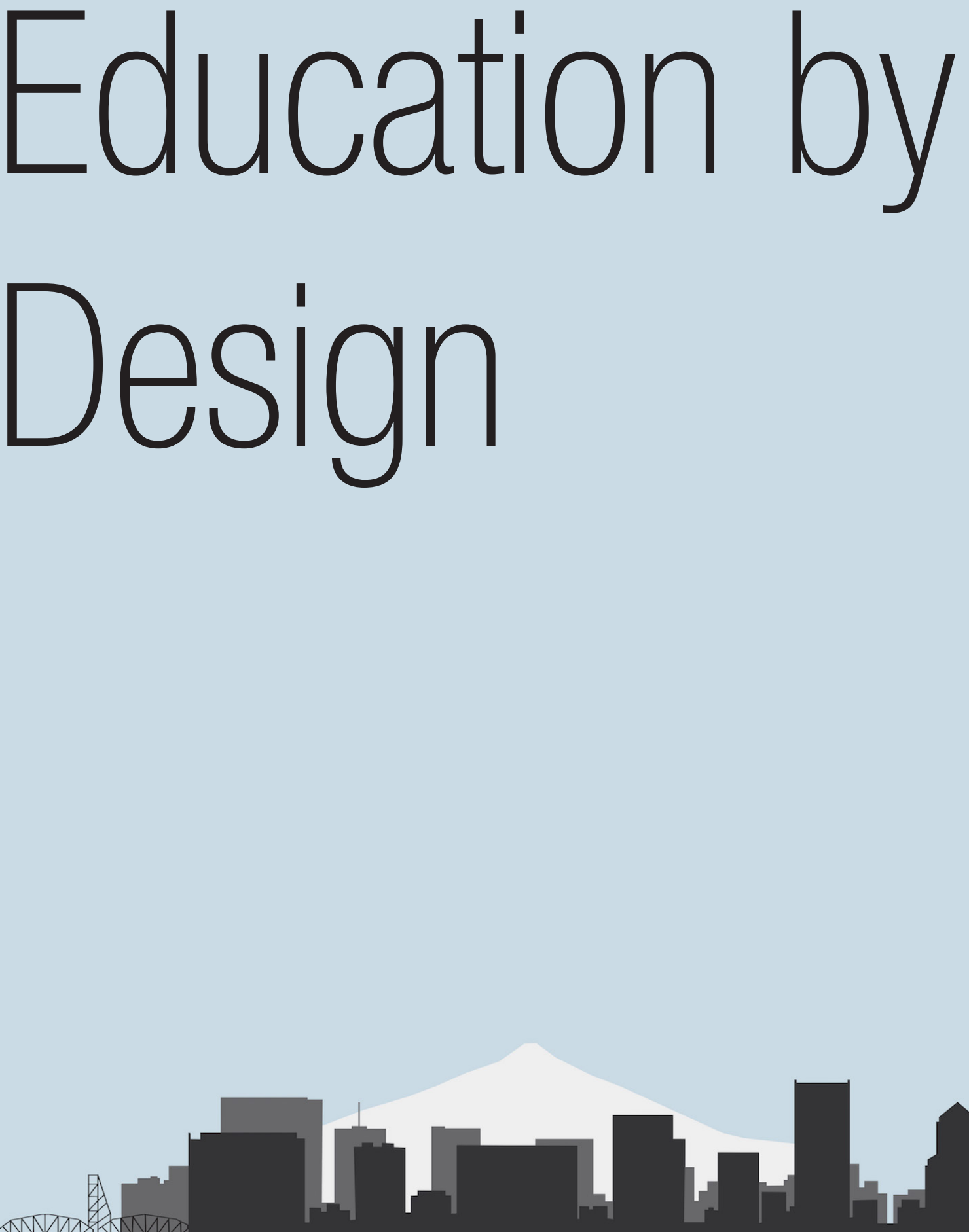

2019 Reynolds Symposium: Education by Design October 18-20, 2019 


\section{Organizing Committee:}

[co-organizers]

Professor Alison Kwok, Ph.D, FAIA, University of Oregon

Emeritus Professor John Reynolds, FAIA, University of Oregon

\section{[Symposium coordinator]}

Isabel Rivera, Ph.D., University of Oregon

Professor Walter Grondzik, P.E., Ball State University

Professor Bruce Haglund, AIA Assoc., University of Idaho

Assistant Professor Emily McGlohn, AIA, Auburn University

Associate Professor Ulrike Passe, lowa State University

Assistant Professor Siobhan Rockcastle, Ph.D., University of Oregon

Sharon Refvem, FAIA, LEED Fellow, Senior Associate and Director, Sustainability Resource Group, Hawley Peterson Snyder 


\title{
Creativity, Calculation, \& Curation: Research as a Framework for Cross-Disciplinary Collaboration
}

\author{
Michelle Laboy \\ Assistant Professor \\ Northeastern University \\ Boston, MA \\ m.laboy@northeastern.edu
}

\author{
David Fannon \\ Assistant Professor \\ Northeastern University \\ Boston, MA \\ d.fannon@northeastern.edu
}

\begin{abstract}
Challenges of building resilience and sustainability in the built environment demand collaboration across multiple disciplines in both research and practice. Traditional academic settings offer fertile but often challenging context in which research faculty can foster interdisciplinary collaboration informed by and contributing to new and more integrated knowledge. This paper presents an example of such a crosscurricular collaboration occurring through qualitative case study research, quantitative analysis, comparison, and communication design culminating in the curation of knowledge in a major public exhibit. Students studying architecture, engineering, art and design are collaborating across multiple courses and semesters to develop the intellectual content, experiential narratives, and physical artifacts that reflect the diverse opportunities and influence of education on the sustainable built environment. Emerging from the faculty's broader inquiry into the architecture of persistence, this project posits durability of buildings as the ultimate measure of sustainability (and by extension, resilience) in architecture. Using quantitative and qualitative methods, researchers developed a theoretical framework for cultural, ecological and technological durability by analyzing interviews and projects. Six architecture students-who first engaged in the topic in a comprehensive studio-became research assistants documenting and analyzing specific precedents as material assemblies and cultural places. Using the resulting documentation, students in an environmental engineering course conducted whole-building Life Cycle Assessments. This body of quantitative and qualitative content feeds a representation course, in which design students examine the role of exhibitions in architectural discourse and develop narratives and objects that communicate material ecologies, assemblies and cultures to a disciplinary, and nondisciplinary audience.
\end{abstract}

\section{INTRODUCTION}

The challenges of building sustainability and resilience in the built environment demand collaboration across multiple disciplines in both research and practice (Laboy and Fannon 2016). Traditional academic settings, with disciplines divided across a hierarchy of groups and departments can be ill-suited to tackle complex problems or answer questions which do not fit neatly within a category. However, universities offer two potential advantages: colocation of diverse expertise and the common activity of teaching. Complex problems like sustainability demand multiple forms of knowledge, and the academic context offers an unusual concentration of deep expertise spanning a diverse range of topics. As researchers typically create and disseminate new knowledge within their own field, areas of overlap with other disciplines remain unexplored due to cultural and pragmatic differences of language, training, funding, research methods, and venues for dissemination. Here, the second critical feature of academic settings, the universal activity of teaching, offers important common ground to bring together diverse scholars. In siloed academic environments, the development of new curricula and courses present unique opportunities not only for improving student learning, but also for the creation of new knowledge by collaborative teams of research faculty. Degree and accreditation requirements, course credits, and schedule differences can still present challenges to the idealized integration of courses from multiple disciplines in the same time and space. This research team explored the potential for interdisciplinary collaboration across multiple courses, faculty, and semesters; advancing ongoing research on Durability as the measure of Sustainability through the integration of topics and methods from different disciplines, 
and the dissemination of this integrated knowledge to the general public in a major exhibit. In this model, the outputs of one course served as the inputs of another, and multiple faculty contributed to each course. By democratizing access to the results for a wide audience, the hybridization and integration of knowledge becomes the shared goal between disciplines. This paper evaluates the student learning outcomes and the advancement of research through interdisciplinary integration between faculty in a cross-curricular model for design education.

\section{Research as bridge between Teaching and Practice}

Calls for greater interdisciplinary collaboration are something of a perennial subject with an endless sense of urgency driven by increasingly complex problems facing future professionals (National Academy of Engineering 2005, 150). Research universities offer a fruitful context for meaningful collaboration because research faculty can leverage shared resources and connect across disciplines through teaching. In spite of this promise, and many efforts and teaching collaborations between architecture and engineering, truly transformative pedagogical models are scarce in the scholarship of teaching and learning, especially models that employ hybrid methods and engage non-experts, usually labeled transdisciplinary (Laboy and Onnis-Hayden 2019). Many limitations occur because teaching seeks to emulate practice, which perpetuates the exact absence of collaboration in practice that the experiment with greater collaboration in school hopes to avoid! Instead, this paper argues for and provides an example of using research as the bridge linking teaching to practice across disciplines while simultaneously promoting greater collaboration between research faculty thanks to the shared common ground of teaching. Teaching design practice allows research faculty to adopt an integrative model in which research informs and is informed by both teaching and practice (Figure 1). The integrated relationships advance all three domains by formulating questions in teaching that interrogate practice, extracting dispersed knowledge from practice, and reorganizing that knowledge as conceptual frameworks and new provocations that advance learning in the academic and professional fields. Just as shared teaching bridges between different researchers, research bridges between teaching and practice.

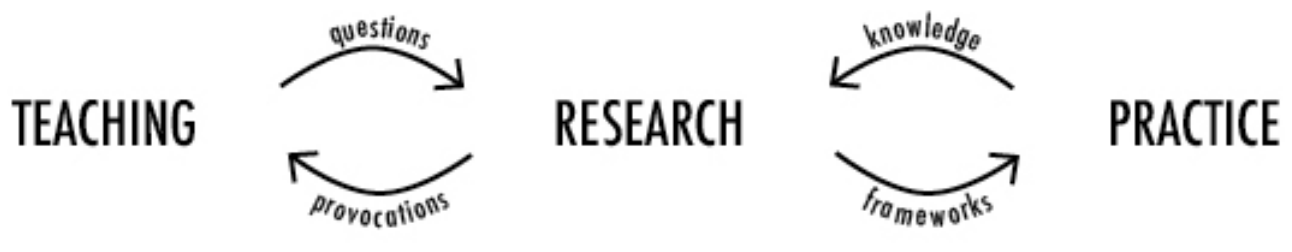

Figure 1: In this model, research acts as a bridge between teaching and practice by formulating questions that can create and disseminate new knowledge in practice, and advance learning

This paper presents an example of applying this model at Northeastern University in Boston. The collaboration between faculty from architecture, engineering, and design emerged from, and advanced research about sustainability of the built environment, while challenging established teaching methods for students across multiple courses. This reflexive integration of research and teaching incorporated design exploration, qualitative case study research, quantitative analysis, and communication design. Students studying architecture, engineering, and design are collaborating across three upper-level undergraduate courses and three semesters to develop the intellectual content, experiential narratives, and physical artifacts that reflect the diverse opportunities and influence of education on the sustainable built environment.

\section{BACKGROUND: INTEGRATING TOPICS AND GOALS}

\section{Future Use Architecture: the teaching origins of the research question}


The research faculty at Northeastern University first conceived of Future-Use Architecture as a way to teach Building Science and Integrated Systems in the design studio, by focusing students on the environmental and cultural performance of systems as generators of the essential qualities of long-lasting and adaptable architecture (Fannon and Laboy 2016). Subsequent revisions expanded the narrow focus using the concept of Resilience to broaden the criteria from simple programmatic adaptation to a wider conception of architecture over time (Laboy and Fannon 2015). The pedagogy produced excellent work year after year: consistently creative and thoughtful concepts for sustainable buildings and sites developed to a high degree of technical resolution. The apparent success of the teaching model prompted some fundamental research questions about architecture beyond the studio, for example, Does thinking about architecture as a long-lasting and adaptable frame for human activity-rather than as a mechanistic solution responding to program-driven requirements-produce better buildings in practice? The researchers hypothesized it might, and as a corollary, wondered if long-lasting buildings are better, what attributes make them better, and what makes them persist?

\section{Regenerative Design and Adaptive Reuse: practice seeking answers from research}

The College of Fellows of the American Institute of Architects awards the Latrobe Prize every two years to a team of researchers with the goal of expanding knowledge on an area of interest to the architecture profession. The call for proposals in 2017 had the topic "Regenerative Design and Adaptive Reuse": (American Institute of Architects College of Fellows 2016). By borrowing an early model of ecological design that imagines the built environment as a living system (Lyle 1994) and by referencing use adaptability, the call expressed the profession's effort to engage with global issues of resilience and sustainability in the face of uncertain change. It also aligned neatly with the questions raised in the Comprehensive Design Studio. Winning this grant offered support from the profession, not only in financial terms, but through access to practices, and practitioners. Clearly architects in practice had some knowledge about issues of long-term building from working on adaptive reuse projects or working with clients that care about the adaptability of buildings over time. However, that knowledge was very likely dispersed, anecdotal, and specific to each architect's or project's context, thus precluding any broader discussion within the discipline. The topic that started as a speculation in a studio curriculum was formalized using grounded theory methods-a systematic form of qualitative research that interrogates practice through interviews and case studies-to produce a theoretical framework for the architecture of persistence. (Fannon and Laboy 2019), The framework of twelve themes or design principles that emerged over two years, nearly fifty interviews, and analysis of one-hundred projects includes the topic of Durability, the subject of the exhibit, teaching, and research collaboration presented in this paper.

\section{Materials and Sustainability: research in the service of the public and society}

In the summer of 2018, the Boston Society of Architects issued a call for proposals for exhibits at the BSA Space in 2020, including one on the topic of Materials and Sustainability, with the goal of helping a diverse audience within and outside architecture learn about how materials in architecture affect sustainability. The Latrobe researchers proposed testing the premise of durability as the prerequisite for, and ultimate measure of architecture's ability to sustain human use. The well-known Shearing Layers diagram (Brand 1994) describes buildings as composed of multiple, mutually-depended and occasionally entangled physical systems with varying life spans. Obviously cycles of construction, repair, replacement, and disposal drive resource consumption and waste production for specific materials. However, beyond the quantities and lifecycle impacts of the materials themselves, the assembly performance and cultural significance of these materials in architecture enable or preclude buildings continued use over time. Thus material durability-especially of the structure and enclosure-enables sustained, and therefore sustainable, architecture. This exhibit offered an ideal opportunity to integrate the pedagogical roots of the research questions, the practice-focused goals of the funders, and the expertise of diverse faculty to expand and instrumentalize the research for the education of students, professionals and the public. The architectural exhibition has a long history in the discipline, and scholars have shown great interest in the medium and process itself, and how the institutions and curators "showcase, mediate, and construct public opinion" and "ultimately affect versions of architectural history" (Betsky et al. 2005) But while 
methods of representation are at the core of architectural education, the exhibition and its curation is rarely considered in that context. As a locus for collaboration, the exhibit allowed the research faculty to experiment with the notion of translation as a primary goal of a collaborative research, teaching and practice.

\section{METHOD: INTEGRATING TEACHING AND RESEARCH METHODOLOGIES}

Examining the cultural, ecological and technological implications of durability demands both quantitative and qualitative methods and metrics. As explained earlier, this topic originated in teaching and the research began as primarily qualitative, seeking a holistic understanding of the physical and cultural phenomena that give rise to long-lasting buildings. The grounded theory method of qualitative research traditionally relies primarily on words and themes coded from interview transcripts. In addition, this project treated built artifacts with equal weight as interviews-not least because relevant examples often emerged from these conversations. As a result, buildings were compared and analyzed through coding of spatial patterns and physical attributes in a graphical method analogous to textual coding. While the comparison of systematic drawings played an important role, these example projects-much like the people interviewed-represent a complex, contingent, and multifarious reality worthy of in-depth individual study typically characterized as a case-study. The shift toward case study method marked a general shift towards mixed methods, and opened the door to cross-disciplinary collaborations framed through the lens of teaching. Figure 2 illustrates the shift from nearly pure qualitative methods to a core of case studies, which became a vehicle for mixed methods, including close readings of artifacts, historical research, and even modeling and simulation. Particularly relevant to this paper is the modeling of material impacts through whole-buiding Life Cycle Assessment.

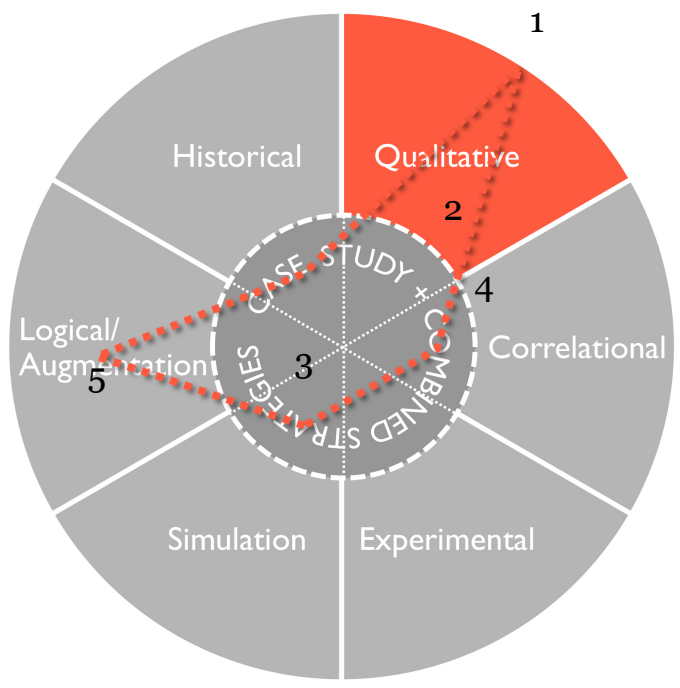

Figure 2: Diagram of architectural research methods (adapted from Groat and Wang 2013, 15) illustrates that while the project began as primarily qualitative research, the introduction of case studies demands and enables further analysis through other research methods, extending the boundaries as shown through the dashed lines and the numeric keys tied to the text below.

In addition to illustrating the range of methods, Figure 2 also provides a helpful frame to discuss the chronology and activities in this research, who was involved, and the relationships among them

1. Grounded Theory: Beginning in summer of 2017, the architecture research faculty conducted over 40 interviews with practitioners, owners and academics. A half dozen of the students in the Future Use Architecture studio course became research assistants, documenting over 100 projects, many of which they had seen in the parallel course on Integrated Building Systems, and 
diagramming them in the same format and scale as case studies. Coding these interviews, and an associated set of projects led to a theoretical framework of Persistence.

2. Qualitative case study research: Faculty and students collaboratively selected four precedent projects to illustrate major architectural materials for the exhibit: wood, brick masonry, steel and concrete. The faculty expanded the interviews to include clients and users of these projects; while students compiled the history of the projects, explored their physical and cultural contexts, collected systems data, and documented their material assemblies.

3. Quantitative analysis: Using the resulting documentation, four teams of students in an environmental engineering course quantitatively analyzed the four precedent buildings through comprehensive, whole-building Life Cycle Assessment (LCA) of each project. The students used the BIM prepared by the architecture students as input for life-cycle inventory. The instructor of this course collaborated with the architecture faculty to design the project and analyze the progress throughout the semester, and became a consultant to the exhibition. The architecture faculty held progress meetings with each team of engineering students to provide input on the specific architectural features to examine, and the research questions to students asked.

4. Comparison, Refinement and standardization of the class data and drawings by a student research assistant enabled a correlational-type study. Unlike a controlled experiment, this work sought to relate the phenomena to the inherent variation among buildings. The faculty hired one engineering student from the LCA class, who worked closely with engineering and architecture faculty to ensure that all four projects were using the same parameters and modeling methods.

5. Logical Argumentation: The body of quantitative and qualitative content became the input for an architectural representation and communication course in which yet another group of students worked with the architecture, engineering and design faculty-including faculty experts in exhibit curation who also joined the exhibition design team - to broadly examine the role of exhibitions in expanding architectural discourse. By developing narratives and objects for the specific exhibit, the students synthesized and translated the interdisciplinary knowledge from the earlier research for a wider audience. The integration and curation of qualitative and quantitative evidence builds the argument for durability as the ultimate measure of sustainability. This argument must be clear but not reductive, acknowledging of the complexity of decisions about material ecologies, assemblies and cultures.

In this curricular experiment colocation and direct interaction between students of different disciplines was not essential as it was more important that each student group had access to research faculty from their own and from other fields. The expectations in each course included clearly-defined aspect of the research problem and specific questions being asked, as well as sufficient context about prior work done by other students, and each students' role in the overall project. The problem was designed as a didactic exercise: to develop new knowledge that could be shared with a wide, non-expert audience about the impact of material decisions on the sustainability of the built environment. This paper presents and evaluates the learning process and outcomes of this curricular model, describes the ways it facilitated interdisciplinary research between faculty, and describes the way it is feeding back into future teaching.

\section{OUTCOMES}

\section{Phasing: Students as novice and expert researchers}

The curricular experiment described here sought to integrate across disciplines, in part by integrating students in each phase of this multi-course, multi-discipline, multi-semester project by helping them understand their work within a larger theoretical framework for more sustainable architectural practice. The staggered schedule allowed faculty to observe students leaving and joining the project at each phase, and each exchange of information included students in two roles: "experts" who were knowledgeable sharing the work they had done in the prior phase, and "novices" approaching that knowledge for the first time. Faculty's systematic observations of these exchanges informed the strategies for teaching and for dissemination to the wider public, and are the basis for the outcomes reported here.

Student motivation increased when they actively and collectively employed their skills in a shared subject, in this case durability. Students building BIM or conducting LCA reported high levels of satisfaction with 
developing their skills, subject knowledge, and contributing to the larger project. In contrast, although the researchers anticipated learning benefits for students transcribing interviews, the solitary, passive, and tedious process proved less valuable for the students and the project than the richness of conversations suggested. Documentation case studies through standard drawings-while also occasionally tedious-used skills linked to students' professional development like interpreting architectural documents and photographs, drawing, and diagramming. Furthermore, the subsequent process of coding drawings as diagrams and developing comparative matrices to identify evaluate certain patterns engaged students in a collective process where their contribution to the research was directly visible.

An example of students as both expert and novice researchers also occurred with the coding of spatial patterns. Student research assistants created base drawings of the case study buildings, a process that required much background investigation and made the students the expert in each particular project. The researcher faculty then proposed a common graphic language to diagram the circulation, infrastructure and human use in relationship to the structural system, which the students deployed. Then, working together, the students and faculty arranged the projects to test and reveal the importance of configuration of these spaces, an example of which is shown in Figure 3. In this instance, the students were experts in specific buildings, but not across arrangements and organization, while the faculty had the broad perspective but did not necessarily know the spatial nuances of each project. This collaboration also led to the decision to select as precedents the purest possible use of structural material, and to use those buildings to reveal material sustainability from ecological, cultural and technological standpoints. Furthermore, by coding and comparing buildings this way, it was possible to select four roughly cubical projects: a form with sufficient formal clarity and compactness to allow easy comparisons across the different material precedents, but containing sufficient differences in spatial strategies, building size and assemblies to reveal the importance of materials.

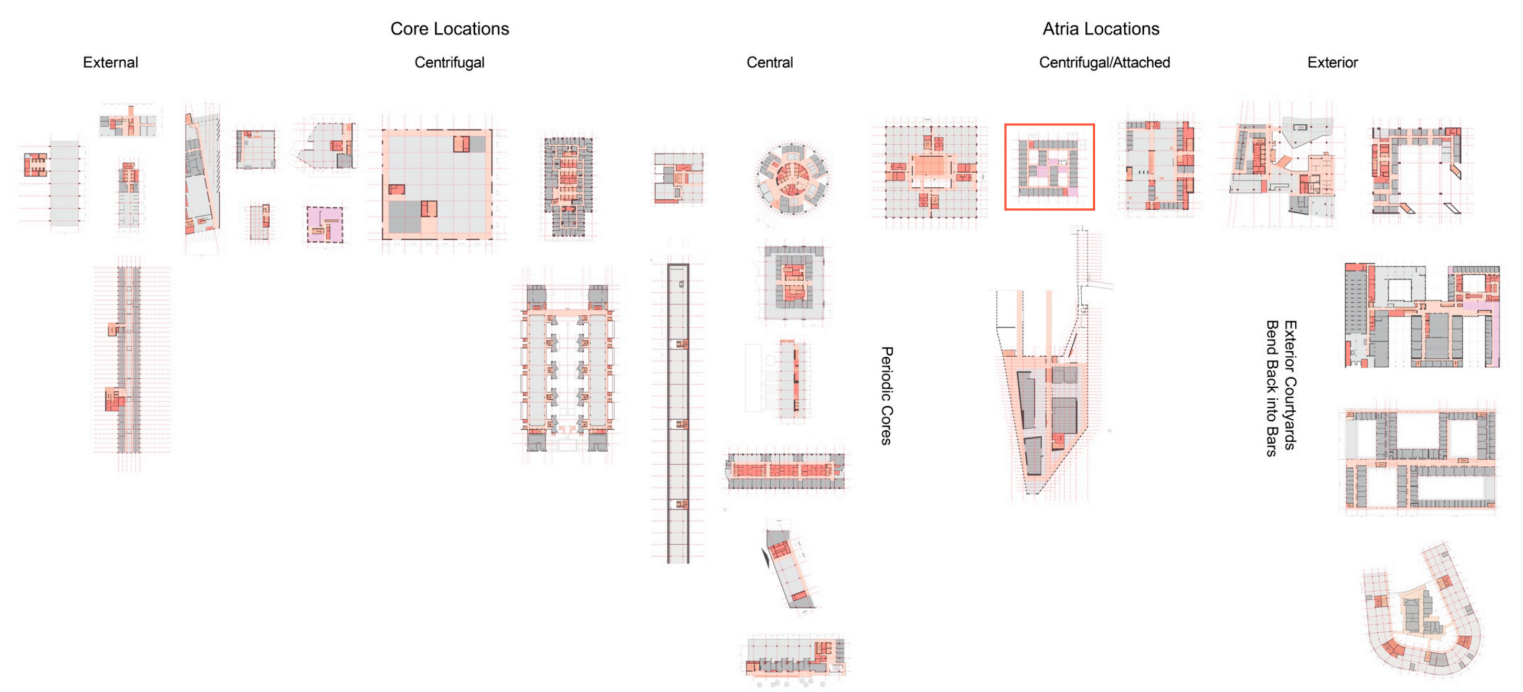

Figure 3: Sample matrix of case studies showing structural pattern organized according to formal strategies for organizing spaces for circulation, infrastructure and human use.

\section{Language: Misalignments of focus and detail}

Every collaboration presents opportunities to build understanding between disciplines, even, and perhaps especially when misunderstandings prompt and deliberate effort to clarify meaning. Existing between multiple disciplines and methods, this experiment resulted in several learning opportunities.

LCA requires a comprehensive bill of materials with a greater scope of information and level of detail than traditional forms of representation in architectural publications and exhibits, but these data seldom arrive ready for analysis in neatly tabular form. In one memorable example, the architecture students 
constructing the Building Information Models of the precedent projects identified the material "Concrete" in assemblies as diverse as composite floors, foundations, precast façade components. The architecture students passed the models along to the engineering students, who blindly used the BIM to take off the quantity of "concrete" for their life cycle inventory without considering the purpose or properties of that material. This mismatch led to a mutual-learning conversation about concrete mixes, finishes, material sourcing, and test procedures. Unlike traditional siloed courses, all students involved in exchanging information between courses benefited from the expanded conversation about the language used to describe materials in different field Furthermore, the architecture students not only learned what parameters matter to LCA experts, but also the nuanced influence of application on material properties, and the need for clear specifications to convey this information. Similarly, the engineering students conducing the LCA developed skills in reading drawings not merely as lines and text, but by interpreting between the lines based on knowledge of construction, history, techniques and precision.

Not all misalignments cross disciplinary lines. The four groups of engineering students included different scope (e.g. foundations or not), used different values for project parameters (e.g. building lifespan), and tabulated different impact metrics (e.g. carbon emissions, eutrophication) when conducing their assessments, thereby preventing any meaningful comparisons across the four precedents. Students had a chance to see and compare results from all four projects in the class presentations, and more than the numeric answers, these presentations highlighted the essential step of defining scope and establishing a common functional unit to enable life cycle calculations useful for analysis. To that end, an interested student from the class was hired as a summer research assistant. She first assembled and standardized the for assessments to ensure they were consistently formatted, included the same scope, and treated study parameters the same way. Once comparisons were possible, she set out to interpret and present the results. In one example, the team tested the effect of treating building lifespan as a variable, rather than as a constant. Changing the unit of analysis from the square meter, to the square meter-year obviously prompts a trade-of between the initial investment and the operating phase, with connections to issues like the time-value of carbon and financial rates of return. More interestingly, this shift prompted additional collaboration. The LCA software assumed brick enclosures last 150 years, which caused a big jump in impact when all the brick was replaced. However, the architects noted that although the masonry project includes brick in the exterior wall assembly, it is covered with a plaster weathering surface which protects the brick but itself requires periodic repair.

Preliminary findings suggest that not only do students learn from each other when the curriculum engages original research, but also that new forms of knowledge can emerge from the interdisciplinary process of data analysis and communication. For example, the concrete floors on the corrugated metal deck completely dominate the LCA impacts of the "steel" building, which complicates and enriches the didactic clarity of precedent buildings chosen based on a single material. The initial desire to for a clear organizational for the exhibit prompted a simple narrative, to which the analysis of quantitative data reintroduced layers of nuance and complexity inherent in architectural practice.

\section{Interpretation: Organization and coherence}

From a teaching and a research perspective, the fact that the exhibit design students did not help develop the research offered an opportunity for the researchers to evaluate how non-expert audiences receive and understand the information. In this case, perhaps the most striking aspect of the student work was a clear interest in the four materials. Many students organized the exhibit content as a series of four independent case studies, one for each material. Given the project's focus on durability, this approach surprised the researchers, who conceived of the exhibit topically, with the four precedents serving only to illustrate those ideas, not as subjects in their own right. The divergence might suggest students found the cohesion inherent to a single building more compelling than that provided by the topics. This result might also suggest a strong material-driven culture in architectural education, at least for these students. Another possible explanation for this is expert blind spot, the concept that expert knowledge includes a rich and multifarious web of connections, while novices-with relatively sparse networks-may struggle to see connections between concepts, and so instead engage them directly and individually. In this case, that would mean the researchers (as experts) selected the precedents only as manifestations of larger ideas, 
but did not make the topical connections sufficiently explicit for non-experts, which has important implications for both teaching and the exhibit.

The work to interpret and communicate knowledge by designing an exhibit in this course also reflects how much the students know (or learned) about the content. The ecological conception of materials seemed to resonate in several alternative organizations. One student chose to arrange the four case studies in a linear narrative sorted by carbon emissions (from wood to concrete). While seemingly simple, this prompted an important conversation about the difference between embodied and operating carbon, and suggests a nuanced narrative is needed however the final exhibit is organized. Working with the products of the research not only develops students' skills in architectural communication, it also introduced them to new knowledge in the field. One student described her exhibit design as "making visible the things you never see" and stated that the story of materials was the most important research finding to share with the public. To that end, she proposed a chronological organization that showed the materials before, during, and after their use in the precedent projects. This arrangement by life cycle stage emphasized the continuity of materials. For all the students, such acts of interpretation represent a higher-level (albeit time-consuming) learning outcome not always available in typical classes that simply "cover the material."

\section{CONCLUSION \& FUTURE WORK}

It is something of a banal cliché to find that improving communication is essential to cross/trans/inter disciplinary work, and it is true that working across disciplines can be a challenge, especially when the different disciplines do not share the same language or research methodologies. Engaging students in combining methods of analysis creates space of shared dialog about teaching, which fosters relationships, improves communication and informs research: simultaneously training students for future practice and expanding the tools and perspectives of the researchers themselves. That said-and without minimizing the importance of communication-these findings are more nuanced than merely getting students to talk to those in other disciplines and providing teachers a venue to talk to other researchers. In this case, the research and teaching process illustrates the importance of the types and format of information exchanged, the understanding of the way different people use the same terms, and the influence of how external audiences interpret the same information. The creation of content and the curation of exhibits proved to be a powerful medium through which to integrate methods, find common language, and expand knowledge.

This cross-curricular model is not the model of ideal integration between students of different disciplines, but it is a good and achievable learning model for students interested in expanding their basic knowledge of integrated design. It is also a good and achievable model for expanding interdisciplinary research among faculty, because it leverages the common ground of teaching, combines knowledge creation and communication, and provides multiple opportunities to disseminate and test the impacts of the work beyond the fields of the individual researchers. As a professional practice and discipline, architectural research is applied research. Translating current architectural research about sustainability into the practice of building sustainable and resilient buildings, requires that knowledge reaches and influences society, as well as architects. Beyond educating future generations of architects to understand and anticipate the consequences of their design decisions in the built environment, they must also communicate outside narrow architectural circles about the value of design research and practice in building a more sustainable environment. The literature repeatedly demonstrates that transdisciplinary research bridges theory and practice, potentially influencing societal decision-making and instruction (Després, Vachon, and Fortin 2011). In a similar way, the work presented in this paper suggests that transdisciplinary teaching-especially if it engages with professionals and the general public-can prompt new and better questions, methods and forms of knowledge from researchers. This paper illustrates that teaching offers a locus to create hybrid methods of research and analysis in the quest to better educate students, as well as to produce, disseminate and apply new knowledge. Future work will need to develop methods to document and analyze the impacts of this work on the audience of the exhibit, and whether new questions emerge from researchers engaging the general public more directly. 
Ultimately, this work closes the cycle or feedback loop between research and teaching, informing ongoing revisions to the Comprehensive Studio and Integrated Building Systems (IBS) courses. In the spring 2020 semester, the courses will occur concurrently with the opening and duration of the exhibit, and several events such as speakers, panels, and reviews will take advantage of the two together. Perhaps most visibility, the thorough understanding and documentation of the precedent buildings makes them the major organizational and didactic tools for teaching Integrated Building Systems, providing critical precedents for each of the four studio phases. The building Haus 2226 in the first phase is the physical manifestation of an argument about the potential of passive systems, of architecture to be structure, enclosure, and comfort systems. The Wood Innovation Center building speaks to broader ecological systems of the second phase, grounded both physically, and within the forest products industry of British Columbia, as it also tries to change that industry, and to slow climate change globally. The ICTA-ICP building, which supports a complex and demanding program of research laboratories and growing spaces through zones of conditioned and semi-conditioned spaces, and gracefully accommodates change, will serve for the third phase exploration of use and active systems. Finally, the carefully-wrought detail of the Salt Lake District Courthouse illustrates the importance of planning and detailing integrated systems of structure, envelope and human use for durable buildings. Naturally no one building contains all aspects of a future-use building, nor is any one precedent the perfect example illustration of the theory of Persistent Architecture, but the quality of interdisciplinary research provides clear didactic illustrations and criteria for effective systems integration, quantitative performance, and qualitative aspects of sustainable and resilient design of the built environment.

\section{ACKNOWLEDGEMENTS}

The authors gratefully acknowledge the collaboration with Professor Peter Wiederspahn (Architecture), Matthew Eckelman (Civil and Environmental Engineering) and Chris Grimley (exhibit design). This work could not have happened without the intellectual contributions and efforts of our students at Northeastern University. The research was funded by the 2017 Latrobe Prize, awarded to David Fannon, Michelle Laboy and Peter Wiederspahn by the American Institute of Architects College of Fellows. The exhibit DURABLE is funded by the BSA Foundation.

\section{REFERENCES}

American Institute of Architects College of Fellows. 2016. “Adaptive Reuse and Regenerative Buildings: Call for Proposals for the 2017-2019 Latrobe Prize.” American Institute of Architects.

Betsky, Aaron, Jeffrey Kipnis, Jeff, Joseph Rosa, Frédéric Migayrou, and Joseph Rosa. 2005. "Exhibiting Architecture: The Praxis Questionnaire for Architectural Curators." PRAXIS: Journal of Writing + Building, no. 7: 106-19.

Brand, Stewart. 1994. How Buildings Learn: What Happens After They're Built. First Edition. New York, NY: Viking Adult.

Després, Carole, Geneviève Vachon, and Andrée Fortin. 2011. "Implementing Transdisciplinarity: Architecture and Urban Planning at Work.” In Transdisciplinary Knowledge Production in Architecture and Urbanism: Towards Hybrid Modes of Inquiry, edited by Isabelle Doucet and Nel Janssens, 33-49. Urban and Landscape Perspectives. Dordrecht: Springer Netherlands. https://doi.org/10.1007/978-94-007-0104-5_3.

Fannon, David, and Michelle Laboy. 2016. “Teaching Building Science in Design Studio.” Journal of the National Institute of Building Sciences, December.

---. 2019. "Methods of Knowing: Grounded Theory in the Study of Future-Use Architecture." In Future Praxis: Applied Research as a Bridge Between Theory and Practice: Journal of Proceedings of the 2019 ARCC International Conference. Toronto: Ryerson University. 
Groat, Linda N., and David Wang. 2013. Architectural Research Methods. 2nd ed. Somerset, UNITED STATES: John Wiley \& Sons, Incorporated. http://ebookcentral.proquest.com/lib/northeasternebooks/detail.action?docID=1166322.

Laboy, Michelle, and David Fannon. 2015. "Learning Comprehensive Building through a Resilience Framework." In Architecture and Resilience on the Human Scale. Sheffield, UK: The University of Sheffield. https://www.sheffield.ac.uk/polopoly_fs/1.504063!/file/Proceedings_27mb.pdf.

---. 2016. "Resilience Theory and Praxis: A Critical Framework for Architecture." Enquiry : The ARCC Journal of Architectural Research 13 (1): 39-53.

Laboy, Michelle, and Annalisa Onnis-Hayden. 2019. "Bridging the Gap between Architecture and Engineering: A Transdisciplinary Model for a Resilient Built Environment.” In Building Technology Educator's Society. Vol. 2019. https://doi.org/10.7275/mr5p-8x02.

Lyle, John Tillman. 1994. Regenerative Design for Sustainable Development. Wiley Series in Sustainable Design. New York: John Wiley.

National Academy of Engineering. 2005. Educating the Engineer of 2020: Adapting Engineering Education to the New Century. Washington, D.C.: The National Academies Press. https://doi.org/10.17226/11338. 